\title{
Epstein-Barr virus detection using gastric biopsy specimens after rapid urease test for Helicobacter pylori
}

\section{다)(1) $(\odot$}

\section{Authors}

Hideo Yanai ${ }^{1}$, Hisashi lizasa ${ }^{2}$, Daisuke Chihara ${ }^{3}$, Tomoyuki Murakami ${ }^{4}$, Jun Nishikawa ${ }^{5}$, Hironori Yoshiyama ${ }^{2}$

\section{Institutions}

1 Department of Clinical Research, National Hospital Organization Kanmon Medical Center, Yamaguchi, Japan

2 Department of Microbiology, Shimane University School of Medicine, Shimane, Japan

3 Department of Gastroenterology \& Hepatology, National Hospital Organization Kanmon Medical Center, Yamaguchi, Japan

4 Department of Pathology, National Hospital Organization Kanmon Medical Center, Yamaguchi, Japan

5 Department of Laboratory Science, Yamaguchi University Graduate School of Medicine, Ube, Japan submitted 11.10.2018

accepted after revision $\quad 27.12 .2018$

\author{
Bibliography \\ DOI https://doi.org/10.1055/a-0859-7233 | \\ Endoscopy International Open 2019; 07: E431-E432 \\ (c) Georg Thieme Verlag KG Stuttgart · New York \\ ISSN 2364-3722
}

Corresponding author

Hideo Yanai, MD, PhD, Department of Clinical Research, National Hospital Organization Kanmon Medical Center, 1-1 Sotoura, Shimonoseki, Yamaguchi, 752-8510 Japan

Fax: +81832411301

yanai.hideo.mu@mail.hosp.go.jp
Epstein-Barr virus-associated gastric carcinoma (EBV GC) is a distinct subtype of GC $[1,2]$.) Many cases of EBV GC have a background of Helicobacter pylori (HP)-positive chronic atrophic gastritis (CAG) [3]. How and when EBV appears in the gastric mucosa is important information for understanding EBV-associated carcinogenesis. Almost all everyone is EBV seropositive, and ordinary serological tests are not useful to detect EBV positivity in gastric mucosa. The pathogenic role of EBV in CAG is unknown. We previously reported that $65.7 \%$ of CAG cases (23 of 35 ) were EBV positive in quantitative PCR (Q-PCR) for gastric biopsy specimens [4]. However, performing research-oriented EBV gastric biopsies for non-cancerous gastritis patients is difficult in esophagogastroduodenoscopy (EGD). On the other hand, the rapid urease test (RUT) for HP is a routine procedure using gastric biopsy specimens [5]. We thought that the gastric biopsy specimen used for RUT might be a useful sample for EBV detection without any additional burden for the patient.

We tested gastric biopsy samples from patients who underwent routine EDG and agreed to EBV detection. They underwent EGD with RUT at the National Hospital Organization Kanmon Medical Center from July through August 2016. RUT was performed using a PyloriTek test kit (Serim Research Corp., United States).

We obtained written informed consent, and this study was approved by the institutional review board. Gastric biopsy samples used for RUT were frozen once and afterword subjected to Q-PCR for the EBV oriP gene. After RUT, a biopsy specimen was quickly collected from the RUT kit and frozen at- $80^{\circ} \mathrm{C}$. Specimens were transported from our hospital to the test laboratory in frozen condition.

EBV detection was successfully performed using two gastric biopsy specimens each after RUT in 10 cases. EBV was detected in four cases (40\%), and HP was detected in nine cases (90\%). Three cases were EBV and HP double positive.

In the current preliminary experience, simultaneous detection of both EBV and HP infection of the stomach using gastric biopsy specimens obtained for RUT was possible and is thus expected to become a useful tool for studying the carcinogenesis of EBV GC. EBV positivity of $40 \%$ is relatively low compared with our former data of $65.7 \%$. However, the current data were obtained from a small number of samples, and we think these preliminary results are indicative of the utility of this method. We will analyze the sensitivity of the current method in a future large-scale study.

Reuse of gastric biopsy specimens for EBV detection after RUT is noninvasive with only minimal difficulty for the endoscopist. We hope our current method will provide a great deal of data on EBV positivity in gastric mucosa and be used for future prevention for EBV GC.

\section{Competing interests}

None 


\section{References}

[1] The Cancer Genome Atlas Research Network. Comprehensive molecular characterization of gastric adenocarcinoma. Nature 2014; 513 $202-209$

[2] Murphy G, Pfeiffer R, Camargo MC et al. Meta-analysis shows that prevalence of Epstein-Barr virus-positive gastric cancer differs based on sex and anatomic location. Gastroenterology 2009; 137: 824 - 833
[3] Yanai H, Murakami T, Yoshiyama $\mathrm{H}$ et al. Epstein-Barr virus-associated gastric carcinoma and atrophic gastritis. J Clin Gastroenterol 1999; 29: $39-43$

[4] Hirano A, Yanai H, Shimizu N et al. Evaluation of Epstein-Barr virus DNA load in gastric mucosa with chronic atrophic gastritis using a real-time quantitative PCR assay. Int J Gastrointest Cancer 2003; 34: $87-94$

[5] Abadi ATB. Diagnosis of Helicobacter pylori using invasive and noninvasive approaches. J Pathog 2018: doi:10.1155/2018/9064952 